\title{
Neopterin, kynurenine and tryptophan as new biomarkers for early detection of rectal anastomotic leakage
}

\author{
Tomas Dusek ${ }^{1,2}$, Julius Orhalmi ${ }^{1}$, Otakar Sotona ${ }^{1}$, Lenka Kujovska Krcmova ${ }^{3,4}$, Lenka Javorska ${ }^{3,4}$, Josef Dolejs ${ }^{5}$, Jiri Paral ${ }^{1,2}$ \\ ${ }^{1}$ Department of Surgery, University Hospital Hradec Kralove, Charles University in Prague, Faculty of Medicine in Hradec Kralove, \\ Czech Republic \\ ${ }^{2}$ Department of Military Surgery, Faculty of Military Health Sciences in Hradec Kralove, University of Defence in Brno, Czech Republic \\ ${ }^{3}$ Research Laboratory at $3^{\text {rd }}$ Internal Gerontometabolic Clinic, University Hospital, Hradec Kralove, Czech Republic \\ ${ }^{4}$ Department of Analytical Chemistry, Faculty of Pharmacy in Hradec Kralove, Charles University in Prague, Czech Republic \\ ${ }^{5}$ Department of Informatics and Quantitative Methods, Faculty of Informatics and Management, University of Hradec Kralove, \\ Czech Republic
}

Videosurgery Miniinv 2018; 13 (1): 44-52

DOI: https://doi.org/10.5114/wiitm.2018.73363

\begin{abstract}
Introduction: At present, there are no strong predictors, nor a useful scoring system, that clearly identifies patients at risk for anastomotic leakage.

Aim: This study aimed to investigate a new method that assesses this risk by monitoring levels of neopterin, tryptophan, and kynurenine, in bodily fluids.

Material and methods: This prospective study included patients who underwent elective rectal resection for carcinoma. The basic condition for inclusion was rectal anastomosis using the double-stapling technique. Preoperative levels of neopterin, tryptophan, kynurenine, and their ratios, were assessed with blood and urine samples. These levels were then monitored for 6 postoperative days in venous blood, urine, and abdominal drainage fluid.

Results: A total of 42 patients were enrolled in the study. Thirty-six patients underwent a laparoscopic resection and 6 patients had an open procedure. No differences were found among neopterin, tryptophan, and kynurenine serum levels. However, the groups were observed to have significant differences in the urinary neopterin/creatinine ratio: the preoperative neopterin/creatinine ratio was $139.5 \mu \mathrm{mol} / \mathrm{mol}$ in the group with leakage, vs $114.8 \mu \mathrm{mol} / \mathrm{mol}$ in the group without complications, $p=0.037$. The same results were observed during the postoperative period, $p=$ 0.012 . Additionally, the group with complications had a higher mean value of neopterin in drainage fluid, $p=0.048$. Conclusions: Our study demonstrated that high preoperative levels of urinary neopterin could be interpreted as a risk for anastomotic leakage. Moreover, pathological levels of neopterin in urine and abdominal drainage fluid could be useful for early identification of anastomotic leakage during the postoperative period prior to its clinical development.
\end{abstract}

Key words: rectal carcinoma, anastomotic leak, neopterin, tryptophan, kynurenine.

\section{Introduction}

Colorectal cancer is one of the most common malignancies in the Czech Republic; approximately 8,000 new cases of colorectal cancer (of which 4,500 occur in men) are diagnosed annually, and roughly 4,000 patients die from it per year [1].

Rectal resection plays a major role in the treatment of rectal cancer. One of the most dreaded

\section{Address for correspondence}

Tomas Dusek MD, Chirurgická Klinika, Fakultní Nemocnice Hradec Králové, Sokolská 581, 50005 Hradec Králové, Czech Republic,

e-mail: dusek.t@email.cz 
complications of rectal surgery is an anastomotic healing complication. Anastomotic leakage $(\mathrm{AL})$ (i.e. leakage of intestinal contents into the surroundings) resulting in pericolic or perirectal abscesses is associated with increased risk of local tumor recurrence and shorter survival, as well as poor functional results [2-5]. The incidence of AL has been reported to range from $2.5 \%$ to $21 \%$ [6-11].

The primary risk factors for $\mathrm{AL}$ include male gender, obesity, duration of surgery exceeding $270 \mathrm{~min}$, greater blood loss, and preoperative chemo/radiotherapy. Low and ultra-low anterior rectal resection are also significant risk factors [2, 6]. In an effort to enable risk prediction and early diagnosis of $A L$, numerous scoring systems have been created and a broad spectrum of various laboratory parameters has been evaluated. Unfortunately, apart from C-reactive protein (CRP) and leukocyte count, other indicators have not been found [12].

One method of predicting anastomotic healing complications prior to clinical manifestation is monitoring the concentrations of neopterin, tryptophan, and kynurenine in bodily fluids.

According to recent literature, evaluation of elevated neopterin levels has not been used to predict postoperative colorectal carcinoma complications.

\section{Aim}

This study aimed to evaluate the potential use of neopterin and other biochemical markers (e.g. kynurenine/neopterin and kynurenine/tryptophan ratios) as predictors of complications associated with anastomotic healing after rectal resection for cancer. The basic working hypothesis is an expected significant increase in these markers, or changes in their ratios within bodily fluids (serum, urine, drainage fluid) in patients with anastomotic healing complications.

\section{Material and methods}

This prospective clinical study included patients who underwent elective rectal resection with primary anastomosis, constructed using the double-stapling technique, at the Hradec Králové University Hospital Surgical Clinic between 1.11.2014 and 30.11.2015. Patients with manually sewn coloanal anastomoses and patients after palliative procedures (i.e. bypass surgery, derivative stoma or probatory laparotomy) were not included in the study.
Patients were selected for the laparoscopic approach based on the patient's overall condition and history of abdominal surgery. Otherwise they underwent an open procedure.

Initial levels of neopterin, tryptophan, kynurenine, and their ratios in serum and urine, were determined from venous blood samples and urine samples taken just prior to surgery. From the $1^{\text {st }}$ to the $6^{\text {th }}$ postoperative day, kynurenine and tryptophan levels, as well as their ratios in serum, urine, and drainage fluid, were monitored until the time of their removal [13, 14]. Also on the $2^{\text {nd }}$ and $5^{\text {th }}$ postoperative days, leukocyte counts and CRP levels in venous blood were assessed. The following additional patient data were analyzed: a) demographic: age, sex, body mass index (BMI), comorbidity index of American Society of Anesthesiologists (ASA) score; b) oncologic: clinical and pathologic tumor staging, tumor grading, tumor invasiveness (presence of angioinvasion, lympho-angioinvasion, perineural invasion), rectal tumor localization; c) therapeutic: completion of neoadjuvant chemoradiotherapy, type of surgical procedure, extent of mesorectal excision, extent of interruption to the arterial supply to the rectum, use of protective ileostomy, procedure radicality; d) clinical: occurrence of $A L$ and its classification [15]. Data were prospectively entered into the ProMed database of patients with rectal cancer.

This study was approved by the Ethics Committee, Hradec Králové University Hospital.

\section{Statistical analysis}

Statistical analyses were performed using SPSS Statistics 22.0 software (SPSS Inc., Chicago, IL, USA). $P$-values $<0.05$ were considered statistically significant. The $\chi^{2}$ test for independence in a contingency table and Fisher's exact test were used.

\section{Results}

A total of 48 patients with rectal cancer, during the previously mentioned period, were enrolled in the study. Two patients were excluded from the study due to having undergone primary treatment with Hartmann's operation, and 4 patients were excluded due to having undergone a manually sewn coloanal anastomosis. Data were analyzed for a total of 42 patients with rectal cancer who underwent a double-stapled rectal anastomosis. Thirty-six patients underwent a laparoscopic resection and 6 pa- 
Table I. Analysis of AL per severity

\begin{tabular}{|lcc|}
\hline Anastomotic leak & $N$ & $\%$ \\
\hline Type A & 7 & 43.8 \\
\hline Type B & 5 & 31.2 \\
\hline Type C & 4 & 25.0 \\
\hline Total & 16 & 100.0 \\
\hline
\end{tabular}

tients had an open procedure. No conversion was found in the analyzed group of the patients.

Of the entire sample, $16(38.1 \%)$ patients had various anastomotic healing complications, and 26 (61.9\%) patients experienced a postoperative course without serious complications.

Type-A AL, not requiring therapeutic intervention, occurred in 7 of 16 (43.8\%) patients. Type-B AL, requiring non-surgical intervention - most frequent-

Table II. Patient demographic data

\begin{tabular}{|c|c|c|c|c|c|}
\hline \multirow[t]{2}{*}{ Parameter } & \multicolumn{2}{|c|}{ Without AL } & \multicolumn{2}{|c|}{$\mathrm{AL}$} & \multirow[t]{2}{*}{$P$-value } \\
\hline & $n$ & $\%$ & $n$ & $\%$ & \\
\hline Sex: & & & & & 0.421 \\
\hline Male & 15 & 57.7 & 12 & 75.0 & \\
\hline Female & 11 & 42.3 & 4 & 25.0 & \\
\hline Age: & & & & & 0.013 \\
\hline Mean & 62.2 & & 68.1 & & \\
\hline Median & 63.5 & & 67 & & \\
\hline Range & $44-75$ & & $58-81$ & & \\
\hline BMI: & & & & & 0.341 \\
\hline Mean & 27.8 & & 27.4 & & \\
\hline Median & 27.8 & & 27.2 & & \\
\hline Range & $21.3-34.6$ & & $22.4-31.9$ & & \\
\hline ASA: & & & & & 0.138 \\
\hline 1 & 1 & 3.8 & 0 & 0 & \\
\hline 2 & 19 & 73.1 & 9 & 56.3 & \\
\hline 3 & 6 & 23.1 & 7 & 43.7 & \\
\hline 4 & 0 & 0 & 0 & 0 & \\
\hline Diabetes mellitus: & & & & & 0.628 \\
\hline Positive & 2 & 7.7 & 2 & 12.5 & \\
\hline Negative & 24 & 92.3 & 14 & 87.5 & \\
\hline Cardiovascular comorbidity: & & & & & 0.940 \\
\hline Positive & 13 & 50.0 & 9 & 56.3 & \\
\hline Negative & 13 & 50.0 & 7 & 43.7 & \\
\hline Pulmonary disease: & & & & & 1.000 \\
\hline Positive & 1 & 3.8 & 0 & 0 & \\
\hline Negative & 25 & 96.2 & 16 & 100.0 & \\
\hline Corticoids: & & & & & N/A \\
\hline Positive & 0 & 0 & 0 & 0 & \\
\hline Negative & 25 & 100.0 & 16 & 100.0 & \\
\hline
\end{tabular}

N/A - not applicable/not available. 
ly rectal lavage or treatment with an Endo-Sponge (B-Braun Medical BV, Melsungen, Germany) - occurred in 5 of 16 (31.2\%) patients. Type-C AL occurred in 4 of 16 (25.0\%) patients, for whom relaparotomy was performed (Table I).

During statistical analyses of AL vs. no complications, no differences in demographic data except age were observed (Table II). The groups were also comparable in terms of oncologic parameters. Likewise, no significant differences in clinical stage, pathologic stage, tumor grade, or even invasiveness (lympho-angioinvasion, perineural invasion), were found. Tumor distribution, according to the location, had borderline statistical significance $(p=0.055)$. Distal rectal cancer was more often seen in the AL group (37.5\%) than in the group without complications (11.5\%). Conversely, 13 of 26 (50.0\%) patients with upper rectal cancer were in the group without complications, while only 3 of 16 (18.8\%) were in the AL group (Tables III and IV). Postoperative course was not influenced by the type of surgery (open/laparoscopic), protective stoma construction, or even procedure radicality. Conversely, results were statistically different regarding mesorectal excisions, $p=0.015$. The total mesorectal excision (TME) was used in $93.7 \%$ (15 patients) in the AL group, and $57.7 \%$ (15 patients) in the group without complications. Not even the extent of arterial interruption influenced $\mathrm{AL}$ in the sample $(p=0.465)$ (Table IV).

The leukocyte count in the group without complications was $8.4 \times 10^{4}$ and in the $A L$ group was

Table III. Data associated with tumors

\begin{tabular}{|c|c|c|c|c|c|}
\hline \multirow[t]{2}{*}{ Parameter } & \multicolumn{2}{|c|}{ Without AL } & \multicolumn{2}{|c|}{$\mathrm{AL}$} & \multirow[t]{2}{*}{$P$-value } \\
\hline & $n$ & $\%$ & $n$ & $\%$ & \\
\hline Clinical stage: & & & & & 0.454 \\
\hline I & 8 & 30.8 & 2 & 12.5 & \\
\hline$\|$ & 2 & 7.7 & 3 & 18.8 & \\
\hline III & 16 & 61.5 & 11 & 68.7 & \\
\hline IV & 0 & 0 & 0 & 0 & \\
\hline Pathologic stage & & & & & 0.298 \\
\hline Complete response: & 4 & 15.4 & 1 & 6.3 & \\
\hline I & 13 & 50.0 & 7 & 43.7 & \\
\hline$\|$ & 3 & 11.5 & 2 & 12.5 & \\
\hline III & 6 & 23.1 & 6 & 37.5 & \\
\hline IV & 0 & 0 & 0 & 0 & \\
\hline Grade: & & & & & 0.066 \\
\hline Well-differentiated & 7 & 26.9 & 0 & 0 & \\
\hline Moderately differentiated & 19 & 73.1 & 15 & 93.7 & \\
\hline Poorly differentiated & 0 & 0 & 1 & 6.3 & \\
\hline Angioinvasion: & & & & & 0.352 \\
\hline Positive & 2 & 7.7 & 3 & 18.8 & \\
\hline Negative & 24 & 92.3 & 13 & 81.2 & \\
\hline Lympho-angioinvasive: & & & & & 1.000 \\
\hline Positive & 3 & 11.5 & 2 & 12.5 & \\
\hline Negative & 23 & 88.5 & 14 & 87.5 & \\
\hline Perineural invasion: & & & & & 0.352 \\
\hline Positive & 2 & 7.7 & 3 & 18.8 & \\
\hline Negative & 24 & 92.3 & 13 & 81.2 & \\
\hline
\end{tabular}


Table IV. Data associated with treatment

\begin{tabular}{|c|c|c|c|c|c|}
\hline \multirow[t]{2}{*}{ Parameter } & \multicolumn{2}{|c|}{ Without AL } & \multicolumn{2}{|c|}{$\mathrm{AL}$} & \multirow[t]{2}{*}{$P$-value } \\
\hline & $n$ & $\%$ & $n$ & $\%$ & \\
\hline Neoadjuvant therapy: & & & & & 0.988 \\
\hline w/o neoadjuvant therapy & 12 & 46.2 & 6 & 37.5 & \\
\hline Chemoradiotherapy & 14 & 53.8 & 10 & 62.5 & \\
\hline Tumor localization: & & & & & 0.055 \\
\hline Upper rectum & 13 & 50.0 & 3 & 18.8 & \\
\hline Middle rectum & 10 & 38.5 & 7 & 43.7 & \\
\hline Lower rectum & 3 & 11.5 & 6 & 37.5 & \\
\hline Type of procedure: & & & & & 0.658 \\
\hline Open & 3 & 11.5 & 3 & 18.8 & \\
\hline Laparoscopic & 23 & 88.5 & 13 & 81.2 & \\
\hline Derivative stoma: & & & & & 1.000 \\
\hline Positive & 7 & 26.9 & 4 & 25.0 & \\
\hline Negative & 19 & 73.1 & 12 & 75.0 & \\
\hline Radicality: & & & & & 1.000 \\
\hline RO & 25 & 96.2 & 15 & 93.7 & \\
\hline R1 & 1 & 3.8 & 1 & 6.3 & \\
\hline R2 & 0 & & 0 & & \\
\hline Extent of mesorectal excision: & & & & & 0.015 \\
\hline Total & 15 & 57.7 & 15 & 93.7 & \\
\hline Partial & 11 & 42.3 & 1 & 6.3 & \\
\hline Dissected vessel: & & & & & 0.465 \\
\hline Superior rectal artery & 21 & 80.8 & 11 & 68.8 & \\
\hline Inferior mesenteric artery & 5 & 19.2 & 5 & 31.2 & \\
\hline
\end{tabular}

$9.8 \times 10^{4}$ on the $2^{\text {nd }}$ postoperative day $(p=0.047)$. The leukocyte count in the group without complications was $6.3 \times 10^{4}$, and in the AL group was $8.6 \times$ $10^{4}$ on the $5^{\text {th }}$ postoperative day $(p=0.010)$ (Table V).

The differences in CRP levels between the groups were more pronounced. On the $2^{\text {nd }}$ postoperative day, the CRP values were $79.1 \mathrm{mg} / \mathrm{l}$ in the group without complications, and $142.4 \mathrm{mg} / \mathrm{l}$ in the $\mathrm{AL}$ group $(p=0.002)$. An even more significant difference was observed on the $5^{\text {th }}$ postoperative day, when the group without complications had a CRP level of $31.8 \mathrm{mg} / \mathrm{l}$, and the $\mathrm{AL}$ group had a CRP level of $151.9 \mathrm{mg} / \mathrm{l}(p<0.001)$ (Table VI).
No statistically significant differences were found among neopterin, tryptophan, and serum kynurenine levels (Table VII).

On the other hand, the urinary neopterin/creatinine (NEO/CREA) ratio was very promising. Prior to surgery, this parameter had a significant statistical difference $(p=0.037)$. The mean NEO/CREA ratio was $139.5 \mu \mathrm{mol} / \mathrm{mol}$ in those who would go on to have $A L$, and $114.8 \mu \mathrm{mol} / \mathrm{mol}$ in those who would go on to have no complications. An even greater statistically significant difference was seen in the average NEO/CREA ratio for the entire observation period; $185.1 \mathrm{\mu mol} / \mathrm{mol}$ in $\mathrm{AL}$ patients $\mathrm{vs}$. 
$142.8 \mu \mathrm{mol} / \mathrm{mol}$ in patients without complications $(p=0.012)$ (Table VIII).

A significant difference in neopterin levels was found when monitoring the parameters within the

Table V. Leukocyte count

\begin{tabular}{|c|c|c|c|}
\hline \multirow[t]{2}{*}{ Day } & \multicolumn{2}{|c|}{ Mean leukocyte count $\left(1 \times 10^{4}\right)$} & \multirow[t]{2}{*}{$P$-value } \\
\hline & Without AL & AL & \\
\hline $2^{\text {nd }}$ & 8.4 & 9.8 & 0.047 \\
\hline $5^{\text {th }}$ & 6.3 & 8.6 & 0.010 \\
\hline
\end{tabular}

drain $(p=0.048)$; this value was $49.03 \mathrm{nmol} / \mathrm{l}$ in the $\mathrm{AL}$ group, and $27.89 \mathrm{nmol} / \mathrm{l}$ in the group without complications. Another parameter that reached borderline statistical significance $(p=0.062)$ was the

Table VI. C-reactive protein (CRP) levels

\begin{tabular}{|lccc|}
\hline Day & \multicolumn{2}{c|}{ Mean CRP level $[\mathrm{mg} / \mathrm{ll}]$} & $P$-value \\
\cline { 2 - 3 } & Without AL & $\mathrm{AL}$ & \\
\hline $2^{\text {nd }}$ & 79.1 & 142.4 & 0.002 \\
\hline $5^{\text {th }}$ & 31.8 & 151.9 & $<0.001$ \\
\hline
\end{tabular}

Table VII. Results for neopterin and kynurenine in sera

\begin{tabular}{|lccccc|}
\hline \multirow{2}{*}{ Parameter } & \multicolumn{2}{c}{ Without AL } & \multicolumn{2}{c}{ With AL } & $P$-value \\
\cline { 2 - 5 } & Average & SD & Average & SD & 0.118 \\
\hline Neopterin [nmol/l] & 9.36 & 2.52 & 18.08 & 8.83 & 0.820 \\
\hline Kynurenine initial $[\mu \mathrm{mol} / \mathrm{l}]$ & 3.96 & 7.09 & 2.65 & 1.38 & 0.108 \\
\hline Kynurenine $[\mu \mathrm{mol} / \mathrm{l}]$ & 2.19 & 0.43 & 2.50 & 0.81 & 0.888 \\
\hline $\begin{array}{l}\text { Kynurenine/tryptophan initial } \\
{[\mu \text { mol/mmol] }}\end{array}$ & 115.38 & 236.96 & 59.80 & 42.93 & 0.089 \\
\hline $\begin{array}{l}\text { Kynurenine/tryptophan } \\
{[\mu \text { mol/mmol] }}\end{array}$ & 51.80 & 16.27 & 59.55 & 18.70 & \\
\hline
\end{tabular}

Table VIII. Results for urinary neopterin

\begin{tabular}{|lccccc|}
\hline \multirow{2}{*}{ Parameter } & \multicolumn{2}{c}{ Without AL } & \multicolumn{2}{c|}{ AL } & P-value \\
\cline { 2 - 5 } & Average & SD & Average & SD & 0.128 \\
\hline Neopterin initial [nmol/l] & 2526.21 & 1268.85 & 4125.06 & 4540.81 & 0.129 \\
\hline Neopterin [nmol/l] & 2583.88 & 1700.87 & 3655.53 & 3083.19 & 0.037 \\
\hline $\begin{array}{l}\text { Neopterin/creatinine initial } \\
{[\mu \mathrm{mol} / \mathrm{mol}]}\end{array}$ & 114.73 & 35.54 & 139.47 & 44.28 & 0.012 \\
\hline $\begin{array}{l}\text { Neopterin/creatinine } \\
{[\mu \mathrm{mol} / \mathrm{mol}]}\end{array}$ & 142.75 & 52.91 & 185.10 & 57.96 & \\
\hline
\end{tabular}

Table IX. Results for neopterin, tryptophan, and kynurenine; and their average levels in drainage fluids

\begin{tabular}{|c|c|c|c|c|c|}
\hline \multirow[t]{2}{*}{ Parameter } & \multicolumn{2}{|c|}{ Without AL } & \multicolumn{2}{|c|}{$A L$} & \multirow[t]{2}{*}{$P$-value } \\
\hline & Average & SD & Average & SD & \\
\hline Neopterin [nmol/l] & 27.89 & 11.31 & 49.03 & 37.41 & 0.048 \\
\hline Tryptophan $[\mu \mathrm{mol} / \mathrm{l}]$ & 71.25 & 21.82 & 76.66 & 29.58 & 0.267 \\
\hline Kynurenine $[\mu \mathrm{mol} / \mathrm{l}]$ & 2.85 & 1.34 & 3.40 & 1.74 & 0.282 \\
\hline $\begin{array}{l}\text { Kynurenine/tryptophan } \\
{[\mu \mathrm{mol} / \mathrm{mmol}]}\end{array}$ & 53.48 & 22.58 & 55.18 & 18.37 & 0.448 \\
\hline $\begin{array}{l}\text { Neopterin/tryptophan } \\
{[\mathrm{nmol} / \mathrm{mmol}]}\end{array}$ & 0.41 & 0.18 & 0.58 & 0.34 & 0.062 \\
\hline
\end{tabular}


neopterin/tryptophan (NEO/TRYP) ratio, which was $0.41 \mathrm{nmol} / \mu \mathrm{mol}$ in patients without complications, and $0.58 \mathrm{nmol} / \mu \mathrm{mol}$ in those with AL (Table IX).

Further statistical analyses found a collective probability of $95 \%$ that the pathologic markers were determinative for predicting the risk of AL. The urinary NEO/CREA ratio was $126.64 \mu \mathrm{mol} / \mathrm{mol}$ prior to the procedure vs. $159.16 \mu \mathrm{mol} / \mathrm{mol}$ after the procedure. Neopterin levels in drainage fluid $>31.53 \mathrm{nmol} / \mathrm{l}$, and NEO/TRYP ratios in drainage fluid $>0.47 \mathrm{nmol} /$ $\mu \mathrm{mol}$, were considered pathological.

\section{Discussion}

In our study, rectal anastomotic leakage was diagnosed in 16 of 42 patients (38.1\%), which is greater than the 2 to $20 \%$ range reported in the literature. This is most likely due to careful monitoring of anastomotic healing at our facility, which stems from rectal anastomotic healing surveillance during 3 time periods: i) prior to the end of surgery; ii) 1 week after surgery, prior to hospital discharge; and iii) 4 weeks after surgery. In this way, we can explain the higher incidence of $A L$ and, in particular, greater detection of clinically silent leaks (type A). For example, a recent publication by Japanese authors reported that their patients had a type $A \mathrm{AL}$ frequency of only $12 \%$, a type B frequency of $52 \%$, and a type C frequency of 36\% [16].

Comparison of the group without a complicated course vs. the AL group showed that the 2 samples had statistically significant differences only with regard to the mesorectal excisions ( $p=0.015)$. Anastomosis at a distance of $<5 \mathrm{~cm}$ from the anus (thus, performed with TME) is a significant risk factor for the occurrence of AL. This is primarily due to extensive dissection in the distal third of the rectum with potential mechanical and thermal damage to its walls, and disruption of the microcirculation. No other statistically significant differences were found between the groups. This shows that, within the presented sample, neither tumor staging nor neoadjuvant therapy (both denoted in the literature as risk factors) influenced AL occurrence.

Activation of the cellular immune response with activation of the monocyte-macrophage system due to bacterial and viral infections, malignant tumors, autoimmune diseases, or antitumor treatment leads to increased neopterin production [2, 17-21]. Afterward, inflammatory response activity can then be monitored via neopterin levels in various bodily fluids. Our study analyzed these levels in serum, urine, and drainage fluid.

It has been shown that higher neopterin levels are present in patients with septic shock than in patients with noninfectious systemic inflammatory response syndrome (SIRS), which reflects inflammatory response activity $[22,23]$. The same results were observed by Ploder et al., during a study in which patients with sepsis or severe trauma had elevated neopterin levels compared to the control group [24].

Baydar monitored differences in urinary neopterin levels in a group of patients with SIRS, sepsis, septic shock, and multiple organ dysfunction syndrome (MODS) and compared them to a control group. The mean neopterin level in the control group was 111 $\pm 11 \mu \mathrm{mol} / \mathrm{mol}$, and $3851 \pm 1081 \mu \mathrm{mol} / \mathrm{mol}$ in the patient group, $p<0.05$. When comparing neopterin levels between individual patient groups, the mean neopterin concentrations were significantly higher in patients with sepsis and septic shock than in those with SIRS [25].

A recent study from the same facility that monitored serum neopterin levels showed that neopterin levels were significantly elevated in patients with sepsis, septic shock, and MODS; and these levels simultaneously correlated with the value of acute physiology and chronic health enquiry (APACHE II) scores. Conversely, the group of survivors had lower serum neopterin levels [26]. In addition to being an inflammatory response marker, neopterin also serves as a significant prognostic factor for sepsis [27].

Our analysis showed that the urinary NEO/CREA ratio prior to the commencement of surgery was significantly higher $(p=0.037)$ in those would go on to be in the AL group vs. those who would recover without complications. In the future, this fact could be used as a major predictor for a high probability of $A L$ and would enable modification of surgical procedures. At-risk patients could undergo protective ileostomy or, in patients with other associated risks (age, sex, radiotherapy, bulky tumors of the distal rectum, distant metastasis), Hartmann's operation is also an option, since it is an effective and safe procedure without a primary anastomosis.

Other statistically significant differences found during the postoperative period included the urinary NEO/CREA ratio $(p=0.012)$, and neopterin levels in drainage fluid from the abdominal drain $(p=0.048)$. The NEO/TRYP ratio in abdominal drainage fluid was 
borderline statistically significant $(p=0.062)$. These results could, perhaps, be used for the basis of early diagnostics for AL (e.g. colonoscopy, CT imaging) as well as timely and effective therapeutic intervention prior to clinical manifestation. Early intervention can protect anastomoses from complications. In type $C$ AL, for example, it would allow for earlier revision of the abdominal cavity with lavage and pelvic drainage, possibly even a protective ileostomy. In type B AL, it would allow for earlier application of local procedures to stop AL and serve as a basis for antibiotic therapy.

On the other hand, our study failed to find any value in monitoring tryptophan, kynurenine, and their ratios. Nonetheless, the results of an Austrian study, which compared tryptophan levels and the kynurenine/tryptophan (KYN/TRYP) ratio in polytrauma patients, showed that tryptophan concentrations decreased in all trauma patients compared to the control group (which had increased KYN/TRYP ratios and kynurenine concentrations) [24]. This is also in line with results from a study by Girgin et al., which showed that the KYN/TRYP ratio unambiguously correlated with sepsis severity [26].

In line with other research [28, 29], our study demonstrated that CRP levels > $140 \mathrm{mg} / \mathrm{l}$ indicate a pathological postoperative course. On the $2^{\text {nd }}$ postoperative day, this value was borderline; but it was unambiguous on the $5^{\text {th }}$ postoperative day. In our sample, there were statistically significant differences in CRP levels between the groups (Table V). Pedersen et al. demonstrated that postoperative CRP levels were significantly elevated in patients with septic complications, and lower in patients with an uncomplicated course. The best cut-off value for CRP as a predictor of septic complications was on the $3^{\text {rd }}$ postoperative day, when CRP levels were $>200 \mathrm{mg} / \mathrm{l}$. This assessment method had a sensitivity of $68 \%$ and a specificity of $74 \%$. The best cut-off value for leukocyte count was on the $2^{\text {nd }}$ postoperative day, with a value $>12 \times 10^{4}$, a sensitivity of $90 \%$, and a specificity of $62 \%$ [30].

Unfortunately, CRP levels (as with other methods based on monitoring blood markers induced by activation of the monocyte-macrophage system) are unable to distinguish AL sepsis from other causes. The $A L$ is the most frequent cause of septic complications after rectal resection with primary anastomosis and it is, therefore, necessary to actively look for this complication when elevated CRP levels are seen.

\section{Conclusions}

Our study has shown that high urinary neopterin levels prior to surgical treatment can be interpreted as a useful biochemical predictor of AL. It makes it relatively easy to identify patients at high risk for AL and modify surgical tactics in terms of the absolute indication for a protective ileostomy to prevent severe septic complications associated with $\mathrm{AL}$ or, in patients with other known risks for primary rectal anastomosis, complete the procedure with Hartmann's operation with a definitive terminal colostomy. At the same, pathological levels of neopterin in urine and abdominal drainage during the postoperative period can be used as an indicator of $\mathrm{AL}$.

\section{Conflict of interest}

The authors declare no conflict of interest.

\section{References}

1. Dusek L, Hoch J, Muzik, J, Pavlík T. Epidemiology and treatment of colorectal carcinoma - Czech population data. Rozhl Chir 2009; 88: 295-302.

2. Portillo G, Franklin ME. Clinical results using bioabsorbable staple-line reinforcement for circular stapler in colorectal surgery: a multicenter study. J Laparoendosc Adv Surg Tech 2010; 20: 323-7.

3. den Dulk M, Marijnen CA, Collette L, et al. Multicentre analysis of oncological and survival outcomes following anastomotic leakage after rectal cancer surgery. Br J Surg 2009; 96: 1066-75.

4. Penninckx F. Anastomotic leakage: a disaster or a challenge with an impact on survival after rectal cancer surgery? Colorectal Dis 2011; 13: 237-8.

5. Jannasch $\mathrm{O}$, Klinge $\mathrm{T}$, Otto R, et al. Risk factors, short and long term outcome of anastomotic leaks in rectal cancer. Oncotarget 2015; 6: 36884-93.

6. Choi DH, Hwang JK, Ko YT, et al. Risk factors for anastomotic leakage after laparoscopic rectal resection. J Korean Soc Coloproctology 2010; 26: 265-73.

7. Matthiessen P, Hallböök O, Andersson M, et al. Risk factors for anastomotic leakage after anterior resection of the rectum. Colorectal Dis 2004; 6: 462-9.

8. Dekker JW, Liefers GJ, de Mol van Otterloo JC, et al. Predicting the risk of anastomotic leakage in left-sided colorectal surgery using a Colon Leakage Score. J Surg Res 2011; 166: 27-34.

9. Brown SR, Mathew R, Keding A, et al. The impact of postoperative complications on long-term quality of life after curative colorectal cancer surgery. Ann Surg 2014; 259: 916-23.

10. Bertelsen CA, Andreasen AH, Jørgensen T, Harling H; Danish Colorectal Cancer Group. Anastomotic leakage after anterior resection for rectal cancer: risk factors. Colorectal Dis 2010; 12: 37-43. 
11. Eriksen MT, Wibe A, Norstein J, et al. Norwegian Rectal Cancer Group. Anastomotic leakage following routine mesorectal excision for rectal cancer in a national cohort of patients. Colorectal Dis 2005; 7: 51-7.

12. Hirst NA, Tiernan JP, Millner PA, Jayne DG. Systematic review of methods to predict and detect anastomotic leakage in colorectal surgery. Colorectal Dis 2014; 16: 95-109.

13. Kujovska Krcmova L, Cervinkova B, Solichova D, et al. Fast and sensitive HPLC method for the determination of neopterin, kynurenine and tryptophan in amniotic fluid, malignant effusions and wound exudates. Bioanalysis 2015; 7: 2751-62.

14. Krcmova L, Solichova D, Melichar B, et al. Determination of neopterin, kynurenine, tryptophan and creatinine in human serum by high throughput HPLC. Talanta 2011; 85: 1466-71.

15. Rahbari NN, Weitz J, Hohenberger W, et al. Definition and grading of anastomotic leakage following anterior resection of the rectum: a proposal by the International Study Group of Rectal Cancer. Surgery 2010; 147: 339-51.

16. Matsuda K, Hotta T, Takifuji K, et al. Clinical characteristics of anastomotic leakage after an anterior resection for rectal cancer by assessing of the international classification on anastomotic leakage. Langenbecks Arch Surg 2015; 400: 207-12.

17. Strohmaier W, Redl H, Schlag G, Inthorn D. D-erythro-neopterin plasma levels in intensive care patients with and without septic complications. Crit Care Med 1987; 15: 757-60.

18. Melichar B, Solichova D, Freedman RS. Neopterin as an indicator of immune activation and prognosis in patients with gynecological malignancies. Int J Gynecol Cancer 2006; 16: 240-52.

19. Melichar B, Solichova D, Svobodova I, Melicharova K. Neopterin in renal cell carcinoma: inhalational administration of interleukin-2 is not accompanied by a rise of urinary neopterin. Luminescence 2005; 20: 311-4.

20. Melichar B, Solichova D, Melicharova K, et al. Urinary neopterin in patiens with advanced colorectal carcinoma. Int J Biol Markers 2006; 21: 190-8.

21. Melichar B, Lenzi R, Rosenblum M, et al. Intraperitoneal fluid neopterin, nitrate, and tryptofan after regional administration of interleukin-12. J Immunother 2003; 26: 270-6.

22. Mitaka C. Clinical laboratory differentiation of infectious versus non-infectious systemic inflammatory response syndrome. Clin Chim Acta 2005; 351: 17-29.

23. Ruokonen E, Ilkka L, Niskanen M, Takala J. Procalcitonin and neopterin as indicators of infection in critically ill patients. Acta Anaesthesiol Scand 2002; 46: 398-404.

24. Ploder M, Spittler A, Kurz K, et al. Accelerated tryptophan degradation predicts poor survival in trauma and sepsis patients. Int J Tryptophan Res 2010; 3: 61-7.

25. Baydar T, Yuksel O, Sahin TT, et al. Neopterin as a prognostic biomarker in intensive care unit patients. J Crit Care 2009; 24: 318-21.

26. Girgin G, Sahin TT, Fuchs D, et al. Tryptophan degradation and serum neopterin concentrations in intensive care unit patients. Toxicol Mech Methods 2011; 21: 231-5.

27. Tasdelen Fisgin N, Aliyazicioglu Y, Tanyel E, et al. The value of neopterin and procalcitonin in patients with sepsis. South Med J 2010; 103: 216-9.
28. Mackay GJ, Molloy RG, O'Dwyer PJ. C-reactive protein as a predictor of postoperative infective complications following elective colorectal resection. Colorectal Dis 2011; 13: 583-7.

29. Almeida AB, Faria G, Moreira H, et al. Elevated serum C-reactive protein as a predictive factor for anastomotic leakage in colorectal surgery. Int J Surg 2012; 10: 87-91.

30. Pedersen T, Roikjær O, Jess P. Increased levels of C-reactive protein and leukocyte count are poor predictors of anastomotic leakage following laparoscopic colorectal resection. Dan Med J 2012; 59: A4552.

Received: 11.10.2017, accepted: 3.12.2017. 